\title{
Erratum
}

\section{First-order quantum-gravitational correction to Friedmannian cosmology from covariant, holomorphic spinfoam cosmology}

\author{
[International Journal of Modern Physics D, Vol. 22, No. 2 (2013) 1350005] \\ Christian Röken \\ Universität Regensburg, Fakultät für Mathematik, \\ 93040 Regensburg, Germany \\ Centre de Physique Théorique de Luminy, \\ Case 907, 13288 Marseille, France \\ christian.roeken@mathematik.uni-regensburg.de
}

Received 29 June 2015

Published 16 July 2015

The imaginary unit on the right-hand side of Eq. (15) should not be there, namely the product $\alpha \beta$ has to be real-valued,

$$
\alpha \beta=-\frac{3 t}{16 \pi \gamma G} \in \mathbb{R},
$$

for real values of the Barbero-Immirzi parameter $\gamma$. As a consequence, and for $\mathrm{d} R / \mathrm{d} T \neq 0$, the first-order quantum Friedmann Hamiltonian constraint (16) becomes

$$
\frac{\mathrm{d} R}{\mathrm{~d} T}-\frac{4 \pi \mathrm{i} l_{\mathrm{P}}^{2}}{R^{2}}=0 .
$$

(Note that for $\mathrm{d} R / \mathrm{d} T=0$, one immediately finds both $H_{\mathrm{Cl}}=0$ and $H_{\mathrm{Qu} .}=0$ (cf. Eq. (13)), leading to the solution $R=$ const. from which one concludes that in this case no quantum correction to the classical scale factor exists.) Since there is no unique way to select a proper cosmological time $T$ for the formal parameterization of the scale factor $R$ from the covariant approach, the canonical parameter choices for the cosmological time are discussed.

First, consider a real-valued cosmological time $T \in \mathbb{R}_{\geq 0}$. The solution of the first-order quantum Friedmann Hamiltonian constraint is easily found by separation of variables and integration with respect to the cosmological time $T$, yielding the solution

$$
R(T)=\left(12 \pi \mathrm{i} l_{\mathrm{P}}^{2} T+\lambda_{0}\right)^{1 / 3} \in \mathbb{C}
$$


where $\lambda_{0}$ denotes a constant. This complex-valued solution is unphysical because a meaningful scale factor is a positive real-valued function and, thus, this solution has to be disregarded. For the remaining, imaginary choices of the cosmological time $T= \pm \mathrm{i} \mathcal{T} \in \mathbb{C}$ with $\mathcal{T} \in \mathbb{R}_{\geq 0}$, the corresponding first-order quantum Friedmann Hamiltonian constraints are given by the differential equations

$$
\frac{\mathrm{d} R}{\mathrm{~d} \mathcal{T}} \pm \frac{4 \pi l_{\mathrm{P}}^{2}}{R^{2}}=0
$$

which can be solved by the same method as in the previous case. These solutions read

$$
R(\mathcal{T})=\left(\mp 12 \pi l_{\mathrm{P}}^{2} \mathcal{T}+\lambda_{0}\right)^{1 / 3}
$$

describing either growing universes with small decelerating expansions of the order $l_{\mathrm{P}}^{2 / 3}$ (positive solution) or shrinking universes with accelerating contractions of the same order (negative solution) with initial values at $R=\lambda_{0}^{2 / 3}$ and cutoffs for the shrinking universe solutions at $R=0$. It is sufficient to study only the expanding universe scenario because the shrinking universe scenario can be obtained by a discrete time reflexion transformation $\mathcal{T} \mapsto-\mathcal{T}$ applied to the latter. Thus, the computations presented in Secs. 3 and 4 are still valid, however, one has to keep in mind that the imaginary cosmological time parametrization $T=-\mathrm{i} \mathcal{T}$ was used, i.e., the scale factor and the derivatives (operator and dot notations) refer to the time parameter $\mathcal{T}$. 